Artículos

\title{
Aplicación de autopsias psicológicas: estudio de caso de dos suicidios en una familia
}

\section{Application of Psychological Autopsy: A Study Case of two Suicides in a Family}

\author{
Karla Patricia Valdés-García'; Hannia Sinahí Ordaz-Cuevas²; Iris Rubí Monroy-Velasco
}

1. Universidad Autónoma de Coahuila

2. Universidad Autónoma de San Luis Potosí

\section{Resumen}

El suicidio es un acto deliberado y complejo, que ocurre a partir de una diversidad de factores. Esta investigación, con enfoque cualitativo, tuvo el objetivo de reconstruir los factores psicosociales asociados al suicidio de dos integrantes de una familia a través del uso de autopsias psicológicas. Se entrevistó a la madre de los occisos sobre factores precipitantes, motivación, letalidad e intencionalidad de los difuntos. Como resultado, se encontró la presencia de precipitantes y motivantes asociados a dificultades de pareja. El método fue ahorcamiento, por lo que la letalidad fue elevada y en la intencionalidad se documentó la ideación suicida previa en un caso. Se concluye que las relaciones de pareja, y rupturas dentro de estas, son un factor significativo, que los suicidios en una familia generan vulnerabilidad en sus miembros a presentar comportamientos suicidas y que se deben continuar estudiando los factores de riesgo para el desarrollo de estrategias preventivas eficaces.

Palabras clave: suicidio, autopsia psicológica, suicidología, factores psicosociales

Karla Patricia Valdés-García. Facultad de Psicología. Universidad Autónoma de Coahuila https://orcid.org/0000-0002-1681-1954

Hannia Sinahí Ordaz-Cuevas. Facultad de Derecho Abogado Ponciano Arriaga Leija. Universidad Autónoma de San Luis Potosí

https://orcid.org/0000-0001-5655-0472

Iris Rubí Monroy-Velasco. Facultad de Psicología. Universidad Autónoma de Coahuila

https://orcid.org/0000-0002-3663-6700

Autor para correspondencia: Karla Patricia Valdés-García karlavaldes@uadec.edu.mx 


\begin{abstract}
Suicide is a deliberate and complex act that can occur due to various factors. The objective of this research was to reconstruct the psychosocial factors associated with the suicide of two family members using a qualitative approach to psychological autopsies. The mother of the deceased was interviewed regarding the precipitating factors, motivation, lethality, and intentionality of the cases. The results identified the presence of precipitants and motivators associated with love difficulties. As the method was hanging, lethality and intentionality were high. Additionally, previous suicidal ideation was documented in one case. It is concluded that couple relationships, and ruptures within them, seemed to be a significant factor, that suicides in a family generate vulnerability in its members to present suicidal behaviors, and that risk factors should continue to be studied to develop effective preventive strategies.
\end{abstract}

Key words: suicide, psychological autopsy, suicidology, psychosocial factors

DOI https://doi.org/10.36793/psicumex.v11i1.404

Recibido 13 de Diciembre de 2020

Aceptado 14 de Agosto de 2021

Publicado 27 de Noviembre de 2021 


\section{Introducción}

El suicidio, declarado un problema de salud pública por la Organización Mundial de la Salud (OMS, 2018a), es un fenómeno complejo y multifactorial. Los comportamientos suicidas se presentan por diversos factores psicológicos, biológicos, sociales y contextuales (Turecki et al., 2019), por ello, se ha analizado desde diferentes disciplinas. Este artículo lo aborda desde la psicología, retomando la suicidología a través del uso de la técnica de autopsias psicológicas.

El suicidio ha estado presente desde la antigüedad, sin embargo, ha variado en función de épocas, culturas y contextos. Se puede conceptualizar desde las locuciones latinas sui y occidere que significan 'matarse a sí mismo' o por su definición como: "el acto autoinfligido para causarse la muerte en forma voluntaria, deliberada, en el que intervienen sucesivamente el deseo suicida, la idea suicida y el acto suicida en sí” (Nizama, 2011, p.82).

Se considera que el suicidio es multifactorial, las razones por las que una persona podría terminar con su vida son muy diversas. Estudios han encontrado que las causas más frecuentes son: trastornos mentales, adicciones, ruptura sentimental, muerte de un ser querido, crisis emocional, enfermedades físicas, desempleo, problemas económicos, experiencias infantiles traumáticas, antecedentes familiares de suicidio y problemas sociales (Nizama, 2011; Clayton, 2018).

De acuerdo con la OMS (2019), ocurren cerca de 800000 suicidios cada año en el mundo, y es la segunda causa de muerte en el grupo etario de 15 a 29 años. Las tasas más elevadas a nivel global se encuentran en el continente europeo con una media de 15.4 suicidios por cada 100000 habitantes, específicamente las tasas más elevadas se presentan en Lituania con 31.9, Rusia 31, Bielorrusia 26.2 y Kazajistán con 22.5.En el continente americano, el promedio de la tasa de suicidios es de 9.8, ocupando los primeros lugares Guyana con 29.2, Surinam 22.8, Uruguay 18.4 y Estados Unidos con 15.3 (OMS, 2019). 
México, en 2018, se posicionó en el lugar veintiuno entre los países del continente americano, con una tasa de 5.1 por cada 100000 habitantes, en donde predominan los suicidios en hombres (8 por cada 100 000) en comparación con las mujeres (2.3 por cada 100 000) (OMS, 2018b). Según el Instituto Nacional de Estadística y Geografía (INEGI), la muerte por suicidio en México de 2010 a 2017 ha reflejado tasas con una tendencia de incremento lento y sostenido (INEGI, 2019; INEGI, 2020).

En el estado de Coahuila en 2017, se registró una tasa de 6.3 muertes por suicidio por cada 100000 habitantes, posicionando al Estado dos lugares por encima de la media nacional (INEGI, 2019). El presente estudio se desarrolló específicamente en el municipio de Saltillo, capital del Estado, ubicado en la región sureste, en dónde en 2018 se registraron 93 suicidios (Instituto Municipal de Planeación Saltillo [IMPLAN], 2018; INEGI, 2019b) y en 2019 se colocó como el séptimo municipio en el país con mayor número de casos (Tun, 2020).

\section{Suicidología y autopsia psicológica}

La suicidología es una ciencia que estudia los procesos autodestructivos; su ícono representativo es el suicidio. Se enfoca en la comprensión y análisis de los comportamientos, pensamientos, actitudes y sentimientos autodestructivos. Los estudios y análisis desde la suicidología están intrínsecamente influidos por nociones socioculturales, que buscan la articulación interdisciplinaria y cuyo objetivo es dar cuenta de los factores biológicos, psicológicos, éticos, sociales y culturales que se van entrelazando en la disposición a comportamientos suicidas en sus diferentes manifestaciones (Martínez, 2017).

Edwin Shneidman, padre y uno de los principales exponentes de la suicidología, buscó comprender el acto suicida desde elementos como la planeación, pensamiento, deseo e intencionalidad. Para Shneidman, el suicidio es el acto consciente de autoaniquilación, que se manifiesta como un malestar pluridimensional en un individuo que percibe a la muerte como la mejor solución para resolver la angustia que experimenta (Chávez y Leenaars, 2010). 
Dentro de esta disciplina, una de las herramientas utilizadas para recabar información es la autopsia psicológica. El término autopsia tiene su origen etimológico en las expresiones auto, que significa 'propio' o 'de sí mismo’, y opsis, que significa ‘vista’ o ‘ver' (Jiménez, 2001). La autopsia psicológica se propuso en el Centro de Investigación de Suicidios a finales de los cincuenta en Estados Unidos a partir de estudios de muertes dudosas. Robert Litman estableció los elementos esenciales para la aplicación, investigación y análisis de la técnica, la cual tiene como objetivo recabar información para entender los factores que fueron detonantes en la muerte de una persona (Jiménez, 2001).

La autopsia psicológica se conceptualiza como "un proceso de recolección de datos del occiso que permite reconstruir su estado mental y su perfil psicológico antes del deceso" (Quintanar, 2007, p. 46). Realiza la reconstrucción retrospectiva de la vida de una persona para generar una hipótesis sobre la causa, forma y motivo de la muerte. Esta técnica indaga factores precipitantes, motivaciones, el método, grado de letalidad y la intencionalidad (Valdés et al., 2021). El proceso de elaboración se da a partir de la identificación de familiares y allegados, de tal modo que se busca caracterizar a la persona que se quita la vida y así considerar los hallazgos para el desarrollo de tratamientos e intervenciones (Constanza, 2016).

En la presente investigación, con base en los antecedentes planteados y haciendo uso de la técnica de autopsias psicológicas desarrollada por la suicidología, se estableció el objetivo de analizar dos casos de suicidio en una familia y poder reconstruir los factores psicosociales, precipitantes, motivación, letalidad e intencionalidad.

\section{Metodología}

La investigación tuvo un enfoque cualitativo con alcance descriptivo (Hernández et al., 2014); naturalista al situarse en el campo y lugar en donde ocurrieron los suicidios a través del uso de visitas y de una entrevista semiestructurada a la madre de los occisos que permitió recabar información, observar y escuchar el relato de la participante en torno a su percepción del suicidio (Rodríguez et al., 1999). 
Los ejes y categorías utilizados en la autopsia psicológica se definieron a partir de la teoría presentada en los antecedentes y, en específico, de las propuestas de Shneidman para indagar las causas del suicidio (Chávez y Leenaars, 2010).

La técnica utilizada se estructuró con base en dos ejes transversales que consideraron los factores psicológicos, refiriéndose a aquellos aspectos que contribuyen al desarrollo de la personalidad, mantenimiento de la salud y bienestar, y la posible etiología de padecimientos psicopatológicos; y los factores psicosociales, para distinguir las influencias, fenómenos sociales, culturales y ambientales que pudieron haber influido en la salud mental y el comportamiento suicida.

Así mismo, en estas autopsias psicológicas se consideraron cuatro categorías: la motivación, considerada como las razones psicológicas para morir arraigadas en el tiempo de vida, las conductas, pensamientos, estilo de vida y personalidad; los factores precipitantes, entendidos como hechos inmediatos o circunstancias que podrían desencadenar la razón última para quitarse la vida; la intencionalidad, que es el nivel inferido de pretensión que tiene una persona para suicidarse; y por último, la letalidad, que se analiza de acuerdo al método utilizado para morir y las consecuencias que conlleva, evalúa también el grado de lucidez en la planeación, preparación y objetivación de la acción autodestructiva (Valdés et al., 2021).

Esta estructura de factores y dimensiones se utilizó por su lógica inductiva para la obtención de resultados generales, y por su orden deductivo, que va de elementos más generales de la vida de la persona hasta aspectos más puntuales del deceso (se presenta en la tabla 1). 


\section{Tabla 1}

Ejes y Categorías de análisis.

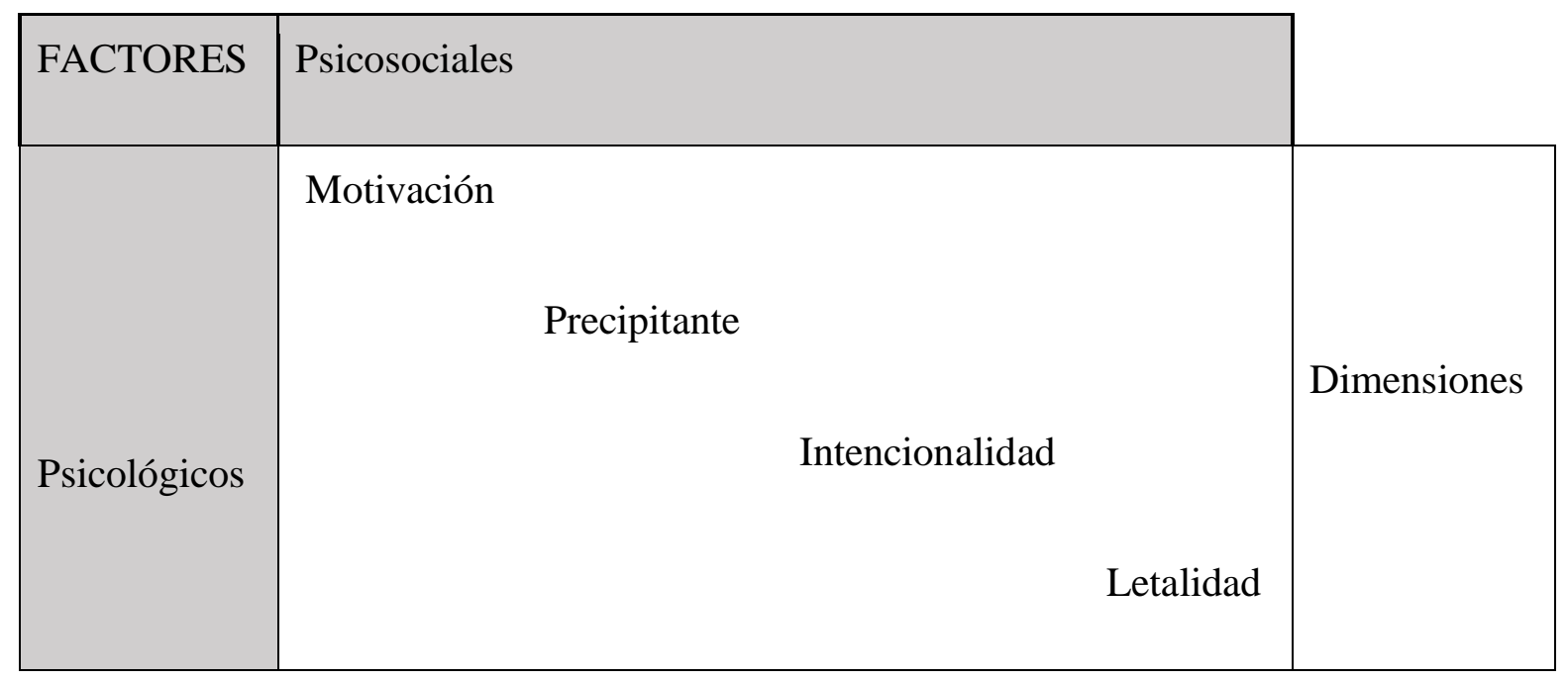

\section{Instrumento}

Se utilizó como técnica principal la autopsia psicológica, se aplicó a la madre de los difuntos, la cual accedió a participar en la investigación y con la información recabada se analizaron aspectos sobre los factores y dimensiones establecidas para el estudio de los dos casos. Para incidir en la validez interna de la investigación, se profundizó y ajustó de forma flexible a los temas abordados por la madre en torno al suicidio de sus hijos.

Sobre los precipitantes, se realizaron preguntas desde la perspectiva de la entrevistada acerca de eventos inmediatos posiblemente relacionados con la decisión de terminar sus vidas, si se observaron cambios en el comportamiento y reacciones afectivas o conductuales ante los posibles eventos estresores. En cuanto a la motivación, se indagó sobre hechos o posibles razones, de cualquier índole y de significancia que pudieron haber fomentado la decisión, así como de la presencia de factores predisponentes tales como una historia familiar de suicidios. En torno a la letalidad, se recabó información sobre el método, lugar, hora del día, posible planeación y acceso a los medios para quitarse la vida. Finalmente, en la intencionalidad se 
cuestionó a la entrevistada si tenía evidencia de que se buscó de manera directa y consciente el suicidio y la detección de conductas, señales o mensajes previos.

\section{Procedimiento y análisis de información}

Debido a las características particulares del caso y del fenómeno estudiado, se utilizó un muestreo no probabilístico, propositivo de participante voluntario (Hernández et al., 2014).Se recogieron los datos en la residencia de la madre, en la cual también se habían llevado a cabo ambos suicidios.

\section{Aspectos éticos}

Se siguieron los lineamientos del Código Ético del Psicólogo de la Sociedad Mexicana de Psicología (2007). La prioridad en todo momento fue proteger la confidencialidad e integridad de la participante, sin dañar su integridad física, moral y/o psicológica.

Se invitó a participar a la madre a través de un organismo gubernamental (Dirección de Atención a Víctimas del Estado de Coahuila), una vez que aceptó, se entregaron los datos de contacto a una de las autoras (K.P.V.G.), quien la contactó en el domicilio de la entrevistada explicando los objetivos, especificaciones y uso de la información, ante lo cual, después de corroborar el uso confidencial de los datos, accedió a participar y estableció una segunda fecha y hora para realizar la entrevista. En el tercer acercamiento, se le compartió el consentimiento informado el cual firmó, y posterior a ello, se dio inicio con la grabación de la autopsia.

Finalmente, se le ofrecieron opciones de atención, canalización y contención emocional en la entrevista, así como a miembros de la familia en caso de que lo requirieran, estas no fueron aceptadas, pues el hermano menor no vivía en la ciudad; el padre no deseaba ser atendido según lo refirió la entrevistada; ya no se tenía contacto con la esposa y nieto para poder ofrecerles el servicio; y la madre ya estaba recibiendo 
atención psicológica y psiquiátrica desde la muerte de su primer hijo, con la cual continuaba hasta la fecha de la aplicación de esta autopsia.

\title{
Participantes
}

Se presentan datos y características específicas de los suicidios en la tabla 2.

\section{Tabla 2}

Datos generales de identificación y del suceso

\author{
Caso 1 Eduardo
}

\begin{tabular}{|c|c|c|}
\hline $\begin{array}{l}\text { Sexo: Hombre } \\
\text { Estado civil: Casado } \\
\text { Ocupación: Empleado } \\
\text { Método: Ahorcamiento }\end{array}$ & $\begin{array}{l}\text { Edad: } 19 \text { años } \\
\text { Religión: católica } \\
\text { Antecedentes suicidas: } \\
\text { Ninguno }\end{array}$ & $\begin{array}{l}\text { Suceso: En noviembre del } 2012 \text {, el } \\
\text { padre encuentra al joven ahorcado en } \\
\text { un patio a un lado de su habitación, } \\
\text { minutos después de una discusión } \\
\text { que sostuvo con su pareja quien se } \\
\text { fue de la casa }\end{array}$ \\
\hline \multicolumn{3}{|l|}{ Caso 2 Miranda } \\
\hline $\begin{array}{l}\text { Sexo: Mujer } \\
\text { Estado civil: unión libre } \\
\text { Ocupación: Empleada } \\
\text { Método: Ahorcamiento }\end{array}$ & $\begin{array}{l}\text { Edad: } 26 \text { años } \\
\text { Religión: católica } \\
\text { Antecedentes suicidas: } \\
\text { Hermano }\end{array}$ & $\begin{array}{l}\text { Suceso: En enero del } 2016 \text {, el padre y } \\
\text { la pareja la encontraron ahorcada en } \\
\text { su habitación con un cable dentro del } \\
\text { closet }\end{array}$ \\
\hline
\end{tabular}

La entrevistada será nombrada como María, y sus hijos, Eduardo y Miranda; todos los nombres fueron modificados por cuestiones de confidencialidad. La madre, de 54 años, tiene formación en una licenciatura del área de la salud, se dedica al hogar, su estado civil es casada, profesa la religión católica y tuvo 3 hijos, de los cuales dos se suicidaron. 


\section{Estructura familiar nuclear}

La composición de la familia nuclear de los occisos estaba conformada por los padres y tres hijos: Miranda, Eduardo e Israel. Eduardo estaba casado y tenía un hijo de un par de meses en el momento de su muerte. La relación que lleva con su esposa, según lo referido por la madre, era problemática, ya que constantemente tenían discusiones. Miranda era la hija mayor y vivía con su pareja en unión libre, no tenían hijos y, al parecer, mantenía una relación conflictiva con su pareja. Ambos hermanos vivían en casa de sus padres con sus respectivas parejas. Israel, por razones académicas, vivía en otra ciudad cuando falleció su hermana mayor.

\section{Descripción de los casos}

Caso 1. Eduardo tenía 19 años cuando se quitó la vida. Él estaba casado, y según refiere la madre, al inicio de su matrimonio vivió con su esposa en casa de sus suegros, al poco tiempo la pareja se fue a vivir a la casa de los padres de Eduardo, en la cual también vivía su hermano menor, quien estudiaba y su hermana mayor quien trabajaba en una empresa.

La madre señaló que Eduardo mantenía una relación de pareja con diversos conflictos, incluso desde el inicio del noviazgo las peleas eran frecuentes y aparentemente estas se intensificaron a partir del nacimiento de su hijo. También comentó que la pareja se separaba y regresaba en repetidas ocasiones. Eduardo, siendo aún menor de edad, contrajo matrimonio quince días antes de que naciera su hijo. En cuanto a sus relaciones familiares, se reportó que mantenía buena comunicación con su madre y hermana, con esta última asistía a reuniones sociales y fue en una de estas donde conoció a su futura esposa; respecto a su padre y hermano menor no se contó con información suficiente para determinar la calidad de la relación.

Además, la madre informó que ella y su hija Miranda no tenían buena relación con la esposa de éste, indicó que su hijo y nuera tenían discusiones frente a su nieto, que en ocasiones Eduardo reaccionaba de manera violenta, destruyendo objetos y/o dando golpes en los muebles, considerando la madre que esta era 
una forma de desahogo por las peleas que tenían, y comentó que nunca vio que su hijo lastimara físicamente a su nuera.

Caso 2. Miranda se suicidó cuatro años después que su hermano, tenía 26, vivía con sus padres y con su pareja en unión libre, tenían poco más de un año de relación. La madre refirió que la relación de la familia con la pareja de su hija era buena, ya que él era una persona que ayudaba con las labores del hogar y ella lo veía como un hijo más.

En cuanto a la relación específica entre Miranda y su pareja, la madre expuso que tenía problemas, porque ella quería casarse y él aparentemente no mostraba interés en el tema. Además, comentó que no compartían pasatiempos ni intereses, no salían juntos a reuniones, fiestas o centros nocturnos, y que su hija solía ir a estos lugares sola o con sus amigas. Miranda tuvo varios intentos de terminar la relación de pareja, pero él le decía "hagas lo que hagas, yo no te voy a dejar”, indicó la madre.

Las interacciones de Miranda con su madre eran cercanas, de confianza y apoyo. En cuanto a la relación con su padre no se cuenta con datos al respecto. Así mismo, la mamá refiere que existían celos hacia el apoyo económico que recibía Israel, su hermano menor, y enojo hacia Eduardo por haberse quitado la vida y abandonar a su hijo.

\section{Resultados}

Los resultados, que a continuación se presentan, están estructurados en función de los ejes y categorías de análisis definidas previamente. Del mismo modo, se contrastan con el sustento teórico que soporta esta investigación.

\section{Factores precipitantes: del riesgo al acto}

A partir de los análisis, se induce que uno de los hechos que pudo precipitar el deceso en el caso de Eduardo fue la discusión que tuvo con su esposa la noche que ella se fue de casa. La madre informó que minutos después de que la esposa de Eduardo se fue con su hijo se escuchó un ruido en el patio, por lo que 
salieron y vieron que Eduardo se había ahorcado. Es por esto que la madre menciona que ese fue el detonante para que su hijo se suicidara.

En este sentido, se encontró que en la relación que mantenían Eduardo y su esposa los problemas al parecer eran constantes. La madre comentó que frecuentemente les decía: “ya déjense sí solamente van a estar peleando" y el occiso respondía que sí, pero nunca lo llevó a cabo. Según la percepción de la madre, su hijo estaba en calma cuando no estaba con su pareja, pero cuando su esposa lo buscaba, él acudía de inmediato, se reconciliaban y continuaban con los mismos conflictos y dinámica familiar.

En cuanto a los factores precipitantes del suicidio de Miranda, María mencionó que la noche de la muerte de su hija, esta había salido de fiesta con sus amigas e ingerido bebidas alcohólicas. Al llegar a su domicilio estaba molesta y tuvo un enfrentamiento con su madre, pues antes de llegar a casa llamó para preguntarle si tenía dinero para pagar un taxi, a lo cual ella le respondió que no, posteriormente la madre le regresó la llamada para decirle que había encontrado algo de dinero, pero cuando llegó no era suficiente para pagar el costo del viaje, lo que originó la discusión entre Miranda y su madre por la falta de apoyo económico, ya que según su opinión si se le daba a su hermano menor.

La madre narra que, según la información que ella tenía, fue en el trayecto de la casa de una amiga — que Miranda fue a dejar después del antro — a su domicilio donde pudo haberse producido la decisión de quitarse la vida. María consideraba esto por una publicación que realizó su hija en ese lapso de tiempo y que decía textualmente "ya valió..." junto con una palabra altisonante que la madre no quiso mencionar; posteriormente, encontraron mensajes en su celular de los que se infiere que sostenía otra relación y en los que donde esa otra persona terminaba la relación que tenía con Miranda.

La madre mencionó que Miranda se veía desesperada en la relación formal que llevaba, pues su hija le había comentado que intentó terminarla anteriormente y su pareja le dijo que no la dejaría. La mamá cree que pudo existir estrés debido a la importancia que su hija le daba a tener éxito en su relación; además de 
identificar las problemáticas sentimentales, había ingerido alcohol y tuvo una discusión con su madre previo al suicidio.

Analizando ambos casos, se encontró que la ruptura de la relación de pareja fue un detonante importante, en este sentido, diversos autores (Nizama, 2011; Jiménez y Cardiel, 2013; Clayton, 2018) mencionan que una de las principales causas por las que se lleva a cabo un suicidio son las dificultades dentro de las relaciones interpersonales. En este caso, las relaciones de pareja aparentemente eran problemáticas, y la madre refirió "ahora, ya conforme a pasado el tiempo, nos hemos dado cuenta que los dos [Eduardo y Miranda] su felicidad la basaron en otras personas". También comentó que sus hijos “esperaban más de ellos [parejas sentimentales]... creían que ellos [parejas sentimentales] eran los que les iban a dar la felicidad y... yo digo que eso fue lo que a ninguno de los dos le funcionó [...]”.

Con base en lo anterior y analizando la información desde un aspecto cognitivo y relacional, se puede inferir que en ambos casos el locus de control era externo, observando un vínculo de pareja dependiente, lo cual es considerado como un factor de riesgo en las conductas suicidas (Urra, 2020).

Otro hecho que pudo ser un facilitador en el caso de Miranda fue el consumo de alcohol. Investigaciones han encontrado la ingesta de alcohol como antecedente del acto suicida; también refieren que las consecuencias sociales y clínicas propias del beber en exceso son factores predeterminantes para cometer un suicidio (Mondragón et al., 2013; Terroba et al., 1986). En general, se considera que el consumo de alcohol puede ser un facilitador del suicidio, influyendo como inhibidor de la capacidad de análisis o como anestésico del malestar emocional.

\section{Motivaciones psicosociales: el papel del contexto}

En cuanto a la estructura y dinámica familiar de origen, estaba conformada por cinco integrantes; madre, padre y tres hijos. La madre tenía estudios profesionales pero nunca ejerció, se dedicó al cuidado de la casa. No se cuenta con datos del padre. El hijo menor estudiaba ingeniería en otro estado al momento del 
suicidio de Miranda. La relación con la madre se reportó como buena, pero no se pudo obtener información completa sobre la relación con el padre.

Miranda era la hija mayor, contaba un trabajo estable en una empresa, tenía estudios técnicos en informática, estaba en una relación de concubinato inestable, vivía con sus padres, tenía redes de apoyo y de amistad tanto sociales como laborales.

Eduardo, segundo hijo del matrimonio, trabajaba en una empresa, estaba casado, su esposa se dedicaba al hogar, se reportó que sostenían una relación conflictiva, tenía un hijo y vivían en casa de los padres de Eduardo. María lo describe como una persona tranquila, trabajador, no consumía sustancias (cigarro y alcohol) ni era frecuente que saliera a reuniones o fiestas.

Se reportó como principal motivación de ambos suicidios la relación de pareja, tanto Eduardo como Miranda tenían antecedentes de dificultades y problemáticas en sus relaciones. Según María, estas tenían un lapso de tiempo causando dolor emocional en sus hijos.

En el caso de Eduardo, resalta la presencia de violencia familiar, manipulación y maltrato que se daba desde el noviazgo. La madre hizo alusión a una posible infidelidad por parte de la esposa de su hijo, inferida a partir de mensajes que ella hacía en redes sociales como: “casada entre semana y solterita el fin de semana... y que en su casa ella llevaba los pantalones y el sancho se los quitaba", siendo estos mensajes origen de discusiones que generalmente terminaban en una separación de dos a tres días, después de los cuales volvían a estar juntos.

En relación con la violencia intrafamiliar, María dijo que nunca vio que su hijo agrediera físicamente a su esposa, aunque ella comentó que él la golpeaba. La mamá comentó: “mi hijo no era agresivo con ella, a lo mejor va a decir usted [refiriéndose a la entrevistadora] era su hijo, pero yo jamás la vi golpeada, yo oía que él golpeaba los muebles", aunque reconoce que "como hijo fue diferente, como esposo quien sabe cómo sería". 
En el caso de Miranda había indicios de dificultades de pareja. Según comentó la madre discutían mucho, que ella tuvo la sospecha de que su hija pudo haber tenido otra relación amorosa a la par, que la pareja formal de Miranda insistía en no terminar la relación y que habían ido a terapia de pareja, la cual abandonó primero la pareja de Miranda y después ella. Además, se observaron otros factores de riesgo para el suicidio como la dinámica en la familia nuclear, dificultades en su situación económica y el consumo de sustancias.

Analizando el contexto económico familiar, se sabe que tenía dos ingresos formales, el del padre y el de Miranda, y que apoyaban económicamente los estudios en otra ciudad del hermano menor. El día del suicidio de Miranda se presentó una discusión entre ella y sus padres por falta de dinero para pagar su traslado en taxi, en dicha discusión se evidenciaron celos hacia el apoyo financiero que tenía su hermano menor. María informó que su hija se estaba estabilizando económicamente, pero al parecer aún tenían ciertas dificultades para cubrir algunos de sus gastos.

En el caso de Miranda, un factor de riesgo particular fue el tener un antecedente de suicidio en la familia. Según reportes de la madre, Miranda estaba enojada con su hermano por terminar su vida sin pensar en las consecuencias "mi hija era lo que decía, que... ¿cómo su hermano no había pensado en su niño? ahora el que está mal es el niño por culpa de él”. Además, su pareja había intentado quitarse la vida, lo cual tuvo un impacto negativo en ella "sucedían cosas que a ella [Miranda] en lugar de admirarlo más, hacían que se decepcionara más, él [pareja] intentó suicidarse aquí [casa de los padres]”.

Uno de los aspectos que, desde la perspectiva de la madre, era aparentemente incongruente con el suicidio de Miranda era su personalidad y características psicológicas. La mamá alude que Miranda tenía rasgos de personalidad extrovertida, que tenía buenas relaciones de amistad, era bromista, simpática, ayudaba a las personas. Además, comentó que expresaba actitudes negativas hacia el suicidio. 


\section{Método y grado de letalidad: el cómo, cuándo y con qué del suicidio}

El método para cometer suicidio en ambos casos fue el ahorcamiento mediante sogas que tenían a su alcance dentro de la casa, un mecate de un tendedero y un cable de conexión eléctrica. En los casos analizados existieron aspectos que hacen suponer que se tomaron medidas para disminuir las posibilidades de ser detenidos en su objetivo de quitarse la vida. Uno de estos elementos fue el horario, los suicidios se realizaron por la noche-madrugada, cuando los demás estaban dormidos; el lugar fue en el interior de la casa, en espacios privados.

En el caso de Eduardo, el día del suicidio se encontraban en casa sus padres, hermanos y un amigo de su hermano menor. El padre lo fue a buscar para pedirle un celular y tras no encontrarlo en su habitación salió al patio lugar donde lo encontró ahorcado. Miranda por su parte, se quitó la vida en su recamara ubicada en el segundo piso de la casa, dentro de un closet, que por no tener la altura suficiente requirió que ella se hincara para lograr su cometido, María comentó que "Miranda planeó su suicidio, porque siempre tenía una llave colocada en la parte externa de la puerta de su recamara y ese día la quitó de ahí; nadie podría entrar, y además, aventó su celular para arriba del techo de su cuarto, aparentemente para esconderlo". En la casa se encontraban sólo sus padres, pues su pareja estaba trabajando. La encontraron por la mañana su pareja y su padre.

\section{Intencionalidad: el para qué de la muerte por suicidio}

En ambos casos, la hipótesis que se tiene con respecto del para qué de sus muertes se puede inducir a partir de las problemáticas que tenían en sus relaciones de pareja. En el caso de Eduardo, la madre refirió que cuando se le dio aviso a su esposa ésta comentó “entonces, ¡sí lo hizo!”, dando a entender que estaba enterada o que él le había comunicado la intención de quitarse la vida.

En otras áreas de su vida y relaciones cotidianas, Eduardo no manifestó señales o alguna intencionalidad suicida. No realizaba actividades de riesgo, no consumía sustancias y su madre solo hizo 
referencia a que tenía poco control sobre sus impulsos, lo que podría indicar un suicidio impulsivo que se dio después de la discusión con su pareja.

En el caso de Miranda, se observan algunas señales, la primera de ellas y de la cual se habló con anterioridad, son las peleas y discusiones con su pareja, el consumo de alcohol y tabaco, y un estado que publicó en una aplicación de celular que decía, “ya valió...” junto con una palabra soez omitida por su madre, este mensaje tal vez reflejaba una advertencia, sin embargo, no existe suficiente claridad para poder interpretarlo así. En ninguno de los dos casos se dejó una publicación, nota o carta póstuma que hiciera referencia directa a la intencionalidad de sus muertes.

\section{Discusión}

El suicidio probablemente seguirá siendo uno de los temas de estudio más complejos, multifacéticos y dinámicos al que se confronten los investigadores de la materia. El seguir analizando el suicidio permitirá aportar evidencias para desarrollar estrategias, métodos, herramientas y protocolos efectivos para su identificación y prevención. Esta investigación buscó obtener datos relevantes para la reconstrucción y análisis de factores psicológicos y sociales a través de la aplicación de la autopsia psicológica.

A pesar de que el objetivo se consiguió en una medida razonable, es importante mencionar una limitación del estudio, que fue la obtención de información a partir de una sola fuente, lo cual introduce un sesgo en los resultados al solo tener una mirada de la vida y personalidad de los casos presentados, por lo tanto, no se logró una saturación de información lo suficientemente robusta.

Así mismo, el uso de las autopsias psicológicas por si misma cuenta con la limitación en el sesgo de los datos obtenidos, pues sin importar el número de informantes que puedan aportar información sobre una muerte por suicidio, nunca será posible tener información precisa sobre las categorías analizadas, pues la perspectiva más importante, la del suicida, no es factible de obtener (Valdés et al., 2021). Estas limitaciones 
no demeritan su aplicación y uso, pues el estudio de los casos de suicidios consumados sigue siendo relevante y significativo para la comprensión de los comportamientos suicidas.

La motivación al suicidio generada a partir de dificultades en las relaciones de pareja y familiares se ha reportado en diversos estudios (Arroyo y Herrera, 2019; Barroso, 2019; Hernández-Vázquez et al., 2016; Martínez y Robles, 2016; Vargas, 2017;). En los casos analizados, según lo reportado por María, la relación de pareja era de gran importancia y a la vez vulnerante para sus hijos, por lo que coincide con lo reportado como un factor que puede incrementar las probabilidades de comportamientos suicidas, sobre todo en las relaciones en las que existe dependencia emocional significativa (Echeburúa, 2015).

Otro factor de riesgo para los comportamientos suicidas es la presencia de violencia familiar y conflictos amorosos (Martínez y Robles, 2016; Vargas, 2017; Arroyo y Herrera, 2019; Barroso, 2019). En los casos analizados se reportó violencia emocional o psicológica a través de insultos, amenazas, indiferencia, celos y chantajes (Consejo Nacional de Población, 2012).

Los casos estudiados coinciden en cuanto al lugar y método más frecuentes en México. De acuerdo con INEGI y la Secretaría de Salud, la mayoría de las personas se suicidan en su residencia por la facilidad de consumar el acto y el acceso a los métodos empleados, lo cual genera un impacto mayor en los miembros del entorno familiar; el método más empleado es el ahorcamiento (Jiménez y Cardiel, 2013; Betancourt, 2008).

La letalidad es uno de los elementos importantes al analizar un suicidio. Específicamente en el caso de Miranda se recabaron datos que muestran ciertos aspectos en cuanto a la determinación de lograr su cometido, como realizarlo en un lugar y momento en donde era poco probable que pudiera ser detenida. También llama la atención el que se hincara para ahorcarse, por lo que se infiere la letalidad e intencionalidad del acto suicida. 
El suicidio es una problemática multifactorial y en los casos presentados se observaron, además de conflictos en las relaciones de pareja, algunos otros factores de riesgo como dificultades en la dinámica familiar nuclear, limitaciones económicas y consumo de sustancias (Arroyo y Herrera, 2019; Barroso, 2019; Clayton, 2018; Hernández-Vázquez et al., 2016; Jiménez y Cardiel, 2013; Martín, 2016; Nizama, 2011; Pérez-Chán, 2015).

En el caso de Eduardo, se reportó que dio aviso de sus intenciones, aunque no se sabe si su amenaza había sido reiterada. En este sentido es importante resaltar la afectación de las creencias y mitos en torno al suicidio, como el pensar que quien lo dice no lo hará, que estos mensajes se hacen por razones distintas al deseo real de morir. Opuesto a esto, se ha reportado que el $75 \%$ de las personas que murieron por suicidio lo habían intentado previamente, y que nueve de cada diez lo habían comentado con alguien (Pérez, 1999). Por ello se requiere trabajar en la concientización y desmitificación de aspectos que dificultan la atención y canalización de personas con intencionalidad suicida.

Otros aspectos aparentemente contradictorios en el suicidio de Miranda son los comportamientos reportados acerca de su postura personal e ideológica contraria al suicidio. Diversos estudios reportan que actitudes negativas hacia el suicidio pueden ser un factor protector de muerte por suicidio (Estrada et al., 2019; Ren 2018; Tan, 2017). En este caso se reporta evidencia contraria a la idea de que una persona que rechaza el suicidio queda exenta de cometerlo.

En esta investigación se analizaron los suicidios de dos hermanos que, con diferencia de cuatro años, se quitaron la vida. Además, se puede mencionar el intento de suicidio de la pareja de Miranda, y unos días posterior a la muerte de ella, su mejor amigo se suicidó. Esto coincide con lo propuesto en relación con las secuelas del suicidio, la conmoción social y emocional que deja en las personas cercanas al suicida (López, 2017; Villar-Cabeza et al., 2018; López, 2019; Orri et al., 2019). Según el Programa Nacional de Prevención 
del Suicidio (2013), se estima que existe un riesgo ocho veces mayor de comportamientos suicidas en personas con antecedentes familiares de suicidio en comparación con la población general.

\section{Conclusiones}

La autopsia psicológica permite una aproximación a ciertos elementos relevantes en los casos de muerte por suicidio. Sin embargo, y como ya se había mencionado, el hecho de que se haga de forma retrospectiva y a través de terceros presenta dificultades particulares inherentes en relación con la consistencia y veracidad. Esta limitación no impide reconocer que es una herramienta que ha logrado aproximarnos a la comprensión de este fenómeno social.

La suicidología es una disciplina muy amplia, que no sólo utiliza esta técnica y no solo aborda los suicidios consumados. Es importante y necesario seguir investigando otros elementos de los comportamientos suicidas como la ideación, el deseo, la planeación, los intentos, las secuelas y muchos aspectos más que son inherentes a la problemática, ya que aún es incierto si el comportamiento suicida funciona como una secuencia, si las personas con ideación, intento y suicidio son grupos con características y factores diferenciados entre sí, entre muchas otras interrogantes que existen en torno al tema.

Así mismo, se requieren investigaciones que aborden el fenómeno desde diferentes áreas del conocimiento y desde perspectivas multi, inter y transdisciplinarias, así como desde diferentes posturas epistemológicas que suministren datos empíricos y científicos, que favorezcan el análisis del suicidio. El estudiarlo de la manera más completa posible permitirá desarrollar herramientas, intervenciones y estrategias más efectivas, ya que la comprensión tiene como fin último el desarrollo de políticas públicas que lleven a estrategias efectivas de prevención.

A partir de los resultados presentados se pueden tomar en consideración ciertos aspectos de utilidad para las intervenciones preventivas universales, selectivas e indicadas. Por ejemplo, el difundir información acerca de que una postura contraria al suicidio no es garantía de no realizarlo, que existe evidencia empírica 
en torno a una serie de factores de riesgo que inciden en la generación de vulnerabilidad, como lo es la historia familiar de suicidios, las dificultades en las relaciones interpersonales, sobre todo las familiares y de pareja, el consumo de sustancias; por mencionar algunos de los más relevantes detectados en el presente estudio.

Finalmente, vale la pena recordar a Emile Durkheim, uno de los más grandes estudiosos del suicidio y que sentó las bases de muchas teorías y trabajos posteriores. Este autor menciona que no son las personas las que se suicidan, sino la sociedad a través de ciertos miembros suyos (citado en Jiménez y Cardiel, 2013). En este sentido, se puede concluir que en el suicidio no se atribuyen culpables, sin embargo, si responsabilidades compartidas en cuanto a su estudio, análisis, detección, comprensión y sobre todo prevención.

\section{Conflicto de intereses}

Las autoras declaran no tener conflicto de intereses.

\section{Agradecimientos}

A la participante de la investigación y las instituciones que permitieron la realización de la investigación.

\section{Referencias}

Arroyo, H. y Herrera, D. G. (2019). Análisis psicosocial del suicidio en personas jóvenes indígenas Bribris. Revista Reflexiones, 98, 7-22.

Barroso, A. (2019) Comprender el suicidio desde una perspectiva de género: una revisión crítica bibliográfica. Revista de la Asociación Española de Neuropsiquiatría, 39, 51 - 66. $\underline{\text { https://scielo.isciii.es/scielo.php?script=sci_arttext\&pid=S0211-57352019000100004 }}$

Betancourt, A. (2008). El suicidio en el Ecuador: un fenómeno en ascenso. Boletín Ciudad Segura 26, 4-9. https://repositorio.flacsoandes.edu.ec/handle/10469/2350 
Chávez, A. M., y Leenaars, A. A. (2010). Edwin S Shneidman y la suicidología moderna. Salud mental 33(4), 355-360. http://www.scielo.org.mx/scielo.php?script=sci_arttext\&pid=S0185-33252010000400008

Clayton, P. J. (2018). Conducta Suicida. Manual MSD, Versión profesionales.

Constanza, C. S., Garcés, S., Díaz, E., Jessica, B. G., Pérez, V. y Aristizábal, M. A. (2016). Autopsia psicológica: una herramienta útil en la caracterización del suicidio. Archivos de Medicina 16(2), 410 - 421. https://www.redalyc.org/pdf/2738/273849945018.pdf

Consejo Nacional de Población (2012). Violencia emocional, física, sexual y económica. http://www.violenciaenlafamilia.conapo.gob.mx/en/Violencia_Familiar/Violencia_emocion al_fsica_sexual_y_econmica

Echeburúa, E. (2015). Las múltiples caras del suicidio en la clínica psicológica. Terapia Psicológica 33(2), 117-126. http://dx.doi.org/10.4067/S0718-48082015000200006

Estrada, C.A., Nonaka, D., Gregorio, E., Leynes, C., Del Castillo, R., Hernández, P. M., Hayakawa, T. y Kobayashi, J. (2019). Suicidal Ideation, Suicidal Behaviors, and Attitudes Towards Suicide of Adolescents Enrolled in the Alternative Learning System in Manila, Philippines A Mixed Methods Study. Tropical Medicine and Health 47(22). https://doi.org/10.1186/s41182-019-0149-6

Guevara, B. y Botega, N. (2003). A Semi-Structured Interview for Psychological Autopsy in Suicide Cases. Brazilian Journal of Psiquiatry 25(4), 212-219. https://doi.org/10.1590/S1516-44462003000400006

Hernández, R., Fernández, C. y Baptista, P. (2014). Metodología de la investigación. McGraw-Hill. Hernández-Vázquez, A., Azañedo, D., Rubilar-González, J., Huarez, B. y Grendas, L. (2016). Evolución y diferencias regionales de la mortalidad por suicidios en el Perú 2004 - 2003. 
Revista Peruana de Medicina Experimental y Salud Pública 33(4) 751-757. https://doi.org/10.17843/rpmesp.2016.334.2562

Instituto Municipal de Planeación Saltillo [IMPLAN] (2018). Análisis sociodemográfico y espacial del suicidio en Saltillo. Gobierno Municipal de Saltillo.

Instituto Nacional de Estadística y Geografía. (2019a). Estadísticas a propósito del día mundial para la prevención del suicidio (10 de septiembre). https://www.inegi.org.mx/contenidos/saladeprensa/aproposito/2019/suicidios2019_Nal.pdf

Instituto Nacional de Estadística y Geografía. (2019b). Suicidios registrados. México. https://www.inegi.org.mx

Jiménez, R. A. y Cardiel, L. (2013). El suicidio y su tendencia social en México: 1990-2011. Papeles de población, 19(77), 205-229. http://www.scielo.org.mx/scielo.php?script=sci $\operatorname{arttext\& pid=S1405-74252013000300012~}$

Jiménez, I. A. (2001). La autopsia psicológica como instrumento de investigación. Revista Colombiana de psiquiatría 30(3), 271-276. http://www.scielo.org.co/scielo.php?script=sci_abstract\&pid=S0034$\underline{74502001000300006 \& \operatorname{lng}=\mathrm{e} \& \mathrm{nrm}=\mathrm{iso} \& \ln \mathrm{ln}=\mathrm{es}}$

López, L. C. (2017). Factores psicopatológicos de riesgo en intentos de suicidio. Ciencias Psicológicas 11(1) 89 - 100. http://dx.doi.org/10.22235/cp.v11i2.1350.

López, L. (2019). Perfil de métodos en intentos de suicidio: tendencias e implicaciones para la prevención, Jujuy, noroeste de Argentina. Ciencias Psicológicas 13(2). http://dx.doi.org/10.22235/cp.v13i2.1872 
Martín, V. (2016). Conducta suicida, protocolo de intervención. International Journal of Developmental and Educational Psychology, 2(1), 233-250. https://doi.org/10.17060/ijodaep.2016.n1.v2.298

Martínez, C. (2017). Suicidología comunitaria para América Latina. Teoría y Experiencias. ALFEPSI.

Martínez, J. y Robles, A. (2016) Percepción de actores sociales sobre la conducta suicida: análisis de contenido a través de grupos focales. Informes Psicológicos, 16(2), 53-68. http://dx.doi.org/10.18566/ infpsicv16n2a04

Ministerio de Salud. (2013). Programa Nacional de Prevención del Suicidio: Orientaciones para su implementación. Minsal. https://www.minsal.cl/sites/default/files/Programa_Nacional_Prevencion.pdf

Mondragón, L., Saltijeral, M. T., Bimbela, A. y Borges, G. (2013). La ideación suicida y su relación con la desesperanza, el abuso de drogas y alcohol. Salud Mental, 21(5), 20-27. http://www.revistasaludmental.mx/index.php/salud mental/article/view/718/717

Nizama, M. (2011). Suicidio. Revista Peruana de Epidemiología, 15(2), 81-85. https://www.redalyc.org/pdf/2031/203122516002.pdf

Orri, M., Galera, C., Turecki, G., Boivin, M., Tremblay, R., Geoffroy, M. y Coté, S. (2019). Pathways of Association Between Childhood Irritability and Adolescent Suicidality. Journal of the American Academy of Child \& Adolescent Psychiatry, 58(1), 99 - 107. https://doi.org/10.1016/j.jaac.2018.06.034

Organización Mundial de la Salud. (2018a). Suicidio. https://www.who.int Organización Mundial de la Salud. (2018b). Panel de visualización de datos de Estadísticas de salud mundial Suicidio. http://apps.who.int/gho/data/node.sdg.3-4-viz-2?lang=en 
Organización Mundial de la Salud (2019) Suicide in the World, Global Health Estimates. https://www.who.int/health-topics/suicide\#tab=tab_1

Pérez, S. A. (1999). El suicidio, comportamiento y prevención. Revista Cubana de Medicina General Integral, 15(2),196-217. http://scielo.sld.cu/scielo.php?script=sci_arttext\&pid=S0864-21251999000200013

Pérez-Chán, M. (2015). Familia multiproblemática como factor en el desarrollo de la ideación e intento suicida en adolescentes. Salud en Tabasco, 21(3), 45-54. https://www.redalyc.org/pdf/487/48745738002.pdf

Quintanar, F. (2007). Comportamiento suicida, perfil psicológico y posibilidades de tratamiento. Pax México.

Ren, Y., You, J., Zhang, X. y Huang, J. (2018). Differentiating Suicide Attempters from Suicide Ideators: The Role of Capability for Suicide. Archives of suicide research, 23(1). https://doi.org/10.1080/13811118.2018.1426507

Rodríguez, G., Gil, J. y García, E. (1999). Metodología de la investigación cualitativa. Málaga: Ediciones Aljibe. ISBN: 84-87767-56-7

Sociedad Mexicana de Psicología [SMP]. (2007). Código ético del psicólogo. Trillas.

Tan, L., Yang, Q., Chen, J., Zou, H. Xia, T. y Liu, Y. (2017). The Potential Role of Attitudes towards Suicide between Mental Health Status and Suicidal Ideation among Chinese Children and Adolescents. Child: care, health and development. https://doi.org/10.1111/cch.12471

Terroba, G., Saltijeral, M.T. y Del Corral, R. (1986). El consumo de alcohol y su relación con la conducta suicida. Salud Pública, 28(5), 489-494.

http://www.revistasaludmental.mx/index.php/salud_mental/article/view/322 
Turecki, G., Brent, D., Gunnell, D., O’Connor, R., Oquendo, M., Pirkis, J. y Stanley, B. (2019). Suicide and Suicide Risk. Nature reviews 5(74). https://doi.org/10.1038/s41572-019-0121-0

Tun, J. (2020). Tendencias del suicidio en México: una mirada desde las estadísticas de mortalidad. Recuperado de: https://cdeunodc.wordpress.com/2020/12/ 04/tendencias-del-suicidio-en$\underline{\text { mexico-una-mirada-desde-las-estadisticas-de-mortalidad/ }}$

Urra, S. (2020) Intervención en crisis en suicidio: protocolo para los consultorios ICPSI. Actualizaciones en Psicoterapia Integrativa, 9, 26-34. https://icpsi.cl/wpcontent/uploads/2020/07/

Valdés-García, K. P., Monroy, I. R. y Cazares, I. M. (2019). Reconstrucción Social y psicológica postmortem de víctimas de suicidios mediante la autopsia psicológica. En Mercado- Rivas Martha X., Sánchez-Aranda Magda L., Pérez-Pimienta Mayra C. Compiladores. Psicología integral al servicio de la humanidad. CNEIP.

Valdés-García, K. P., Sánchez-Loyo, L.M., Monroy, I. R. y Carreón C. (2021). Psychosocial Suicide Risk Factors. Gaceta Médica de Caracas, 129(supl 1), 100-109, DOI: http://saber.ucv.ve/ojs/index.php/rev_gmc/article/view/22279/144814488553

Vargas, A. (2017). El suicidio en el ámbito comunitario. Revista Reflexiones, 96(1), 27-39. https://dialnet.unirioja.es/servlet/articulo?codigo=6124745

Villar-Cabeza, F., Esnaola-Letemendia, E., Blasco-Blasco, T., Prieto-Toribio, T., Vergé-Muñoz, M., Vila-Grifoll, M., Sánchez-Fernández, B. y Castellano-Tejedor, C. (2018) Análisis dimensional de la personalidad del adolescente con conducta suicida. Actas españolas de psiquiatría, 46(3), 104-111. https://www.actaspsiquiatria.es/repositorio/20/113/ESP/20-

\section{3-ESP-104-11-630668.pdf}

Cómo citar este artículo: Valdés García, K. P., Ordaz-Cuevas, H. S. ., \& Monroy-Velasco, I. R. . (2021). Aplicación de autopsias psicológicas: estudio de caso de dos suicidios en una familia : estudio de dos suicidios en una familia. Psicumex, 11(1), 1-26, e404. https://doi.org/10.36793/psicumex.v11i1.404 